\title{
Tuberculosis and Pott's disease, still very relevant health problems
}

\author{
Ferran Pellisé
}

Accepted: 30 September 2012/ Published online: 9 October 2012

(C) Springer-Verlag Berlin Heidelberg 2012

Due to a resurgence of tuberculosis (TB) globally, the world health organization (WHO) took an unprecedented step in 1993 and declared TB a global emergency [1]. New challenges, such as worsening of the HIV epidemic and rising TB/HIV co-infection, emergence of drug-resistant TB strains, and poor healthcare access of vulnerable populations proved that the efforts taken since 1995 did not suffice for effective TB control.

Nowadays, TB remains a considerable health burden in the WHO European region [2]. Multidrug-resistant (MDR)$\mathrm{TB}$, a man-made phenomenon, is a major issue in this region, with increasing percentages of MDR-TB among all TB cases documented over the last 10 years [2]. Only one of ten MDR-TB patients receive appropriate treatment: poor healthcare access and poor implementation of fast, effective diagnostic methods for resistance detection hinder proper treatment of this condition [3]. The increasing incidence of MDR-TB has highlighted the insufficiency of histo-pathological diagnosis and pointed to the need for microbiological diagnosis associated with sensitivity evaluation of the causal strain. New improved culture media and identification methods reducing the time required for identification and sensitivity analyses might be extremely helpful [4].

In 2006, the WHO developed a new comprehensive strategy for TB control, the Stop TB Strategy, designed to address the spread of TB and HIV co-infection and MDRTB. The aim of the Stop TB Strategy is to halt the epidemic and reverse it by 2015, with the ultimate goal of eliminating TB as a public health problem by 2050 [5]. Spinal

F. Pellisé $(\bowtie)$

Spine Unit, Servicio de COT, Hospital Vall d'Hebron,

Pg. Vall Hebron 119-129, 08035 Barcelona, Spain

e-mail: 24361fpu@comb.cat tuberculosis, known as Pott's disease, is reported together with other extra-pulmonary TB cases to the joint WHO/ ECDC surveillance system and, therefore, the exact magnitude of this disease is unknown [2]. There remains a need to further analyze the related data by country, to document the extent of Pott's disease, and to develop specific guidelines for timely diagnosis and treatment of this condition.

Anti-TB drug therapy is the mainstay of treatment for spinal TB. However, with destruction of the anterior column of the spine by the infective process, kyphosis may result from progressive anterior collapse. After healing of the spinal infection, the post-TB kyphosis in adults is generally static, but in children kyphosis progresses dramatically in almost $40 \%$ of cases [6]. The medical research council (MRC) Working Party on TB of the spine, which was formed in 1963, designed prospective multicenter clinical trials that ultimately proved that the Hong Kong operation (radical debridement and strut grafting) produces the best short-, medium-, and long-term results in aspects such as fusion rate, spinal deformity, and relapses of abscess and sinus [7]. We now know that progressive bone destruction with severe kyphosis can still develop in patients who have received successful non-surgical medical treatment. These late complications commonly present more than 15 years after the initial spinal infection and suggest that the 15-year follow-up study by the MRC may be insufficient to reach valid long-term conclusions [8].

There is still a high incidence of neurological involvement in Pott's disease, with rates of 10 to $20 \%$ of cases in highly developed nations and 20 to $40 \%$ in underdeveloped countries. Most spinal cord injuries appear in the early, acute phase of the disease. However, patients treated with drugs alone or with simple debridement without fusion may experience disease reactivation, severe 
kyphosis, or late instability, which in turn may lead to lateonset Pott's paraplegia, back pain, sagittal imbalance, and compromised pulmonary function, which can be difficult and risky to treat [8]. Correction of late deformity is only feasible with demanding three-column spinal osteotomies, and posterior vertebral column resection is probably the treatment of choice in extreme kyphosis [9]. Prevention of severe spinal deformity at an early stage should therefore be the modern standard of treatment especially for highrisk patients.

The present ESJ supplement provides a unique, comprehensive overview of the current status of tuberculosis and Pott's disease, considering many of the most relevant epidemiological, diagnostic, and therapeutic aspects. Indepth study of its content, delivered by worldwide experts in the field, should serve to improve our understanding of this condition and the care for affected patients.

We wish you fruitful and enjoyable reading!

\section{Conflict of interest None.}

\section{References}

1. World Health Organization (1994) Global Tuberculosis Programme. Framework for effective tuberculosis control. Geneva, Switzerland. WHO/TB/94.179
2. Dara M, Dadu A, Kremer K, Zaleskis R, Kluge HH. Epidemiology of tuberculosis in WHO European Region and public health response. Eur Spine J. 2012 May 8. (Epub ahead of print)

3. Millet JP, Moreno A, Fina L, Del Baño L, Orcau A, de Olalla PG, Caylà JA. Factors that influence current tuberculosis epidemiology. Eur Spine J. 2012 May 8. (Epub ahead of print)

4. Colmenero JD, Ruiz-Mesa JD, Sanjuan-Jimenez R, Sobrino B, Morata P. Establishing the diagnosis of tuberculous vertebral osteomyelitis. Eur Spine J. 2012 May 11. (Epub ahead of print)

5. European Centre for Disease Prevention and Control/WHO Regional Office for Europe (2012) Tuberculosis surveillance and monitoring in Europe, report 2012. European Centre for Disease Prevention and Control 2012, Stockholm

6. Rajasekaran S (2001) The natural history of post-tubercular kyphosis in children: radiological signs which predict late increase in deformity. J Bone Joint Surg 83B:954-962

7. Mak KC, Cheung KM. Surgical treatment of acute TB spondylitis: indications and outcomes. Eur Spine J. 2012 Aug 16. (Epub ahead of print)

8. Cheung WY, Luk KD. Clinical and radiological outcomes after conservative treatment of TB spondylitis: is the 15 years' followup in the MRC study long enough? Eur Spine J. 2012 May 8. (Epub ahead of print)

9. Boachie-Adjei O, Papadopoulos EC, Pellisé F, Cunningham ME, Perez-Grueso FS, Gupta M, Lonner B, Paonessa K, King A, Sacramento C, Kim HJ, Mendelow M, Yazici M. Late treatment of tuberculosis-associated kyphosis: literature review and experience from a SRS-GOP site. Eur Spine J. 2012 May 25. (Epub ahead of print) 